\title{
Apps para la innovación docente en Criminología
}

\section{Lorea Arenas García ${ }^{\mathrm{a}}$, Ana Isabel Cerezo Domínguez}

${ }^{a}$ Docente investigadora en formación de la Universidad de Málaga (lorea@uma.es), ${ }^{b}$ Profesora Titular de Derecho penal y Criminología de la Universidad de Málaga (aicerezo@uma.es).

\begin{abstract}
:
The appearance of mobile internet devices or smartphones has revolutionized the way we interact with our environment. Thanks to the new abilities of these software applications or Apps, it is now possible to perform multiple tasks simultaneously under a limited budget without geographical barriers. This study aims to identify their advantages and applications in the studies of Criminology, in order to reinvent the qualitative research in educational activities. The expected results allow to anticipate a development of the students skills related to ICT management, in their academic training, the design of research projects and the improvement of scientific reports.
\end{abstract}

Keywords: ICT, Apps, educational innovation, methodology, qualitative techniques, digital skills.

\begin{abstract}
Resumen:
La aparición de dispositivos móviles con internet o smartphones han revolucionado el modo de interactuar con nuestro entorno. Gracias a las prestaciones de las aplicaciones de software o Apps es posible llevar a cabo múltiples tareas de forma simultánea sin barreras geográficas y escaso presupuesto. El presente estudio tiene como objeto identificar sus ventajas y aplicaciones en los estudios de Criminología de cara a reinventar la investigación cualitativa en la acción docente. Los resultados esperados permiten anticipar un desarrollo de las competencias de los alumnos con relación al manejo de las TIC en su formación académica, en el planteamiento de proyectos de investigación y en la elaboración de informes cientificos.
\end{abstract}

Palabras clave: TIC, Apps, innovación docente, metodología, técnicas cualitativas, competencias digitales. 


\section{Introducción}

En la última década la aparición de dispositivos móviles con internet o smartphones han revolucionado la forma de interactuar con la realidad que nos rodea. El envío y recepción de información a tiempo real desde cualquier ubicación transforma el significado de las relaciones y reporta funcionalidad a los procesos de comunicación tradicionales. De esta forma, es posible utilizar el móvil o la tablet para recibir noticias, conversar, participar en redes sociales, comprar y vender, buscar empleo, aprender, etc. En definitiva, llevar a cabo múltiples tareas y gestiones con la ayuda de un dispositivo y la instalación de aplicaciones de software o Apps tan variadas y versátiles como las necesidades a las que responden. A lo anterior, se suma la presencia de un mercado centralizado en el que cualquier usuario puede crear y ofertar su App potenciando su expansión, sobre todo en el sector del ocio y entretenimieto.

No obstante, otros muchos ámbitos, tales como la docencia y la investigación, no son ajenos a las prestaciones que las apps brindan para transmitir conocimientos ${ }^{1}$, organizar las clases y tareas de los profesores ${ }^{2}$, así como ejecutar una investigación empírica con los estudiantes. En este último aspecto se centra el presente trabajo, en las prestaciones que ofrecen las apps para reinventar la investigación cualitativa en el marco de la acción docente. Se trata de un tema muy novedoso escasamente abordado en nuestro país ${ }^{3}$ en las ciencias sociales y del que no se encuentran precedentes en el campo de la educación en criminología ${ }^{4}$.

\subsection{Tipos de Apps}

Todas las aplicaciones de software permiten al sujeto -ya sea profesor o alumnorecopilar información sobre sus propias percepciones o las de otros individuos en relación a un tema en particular, si bien pueden disntinguirse entre aquellas aplicaciones genéricas, utilizadas por diversas disciplinas científicas que hacen uso de técnicas cualitativas, sobre todo de métodos etnográficos (Álvarez, 2008), de las que son especializadas o creadas ad hoc para recabar información de temas concretos de investigación, tales como el miedo al delito y la criminalidad presente en ciertos barrios o zonas.

\footnotetext{
${ }^{1}$ Los apps sirven para aprender, entrenar la memoria, mantener activo nuestro cerebro, organizar nuestro tiempo de estudio, etc. Prueba de ello son la creación de apps como: Luminosity, Goconqr, Selfcontrol, Studious, Babylon, iRae, Khan Academy, Mindomo, etc.

${ }^{2}$ Entre ellas destacan: Remind101, Teacher Aide, TED, Educreations, Nearpod, Viper, Attendance, Box, Trello, etc.

3 En el ámbito comparado cada vez es más frecuente la utilización de las apps en la docencia e investigación. Véanse: García y otros (2015) y Sugie (2016).

4 En España las apps educacionales en criminología se identifican con la descarga de material didáctico y glosarios. Véase: http://noticias.universia.es/educacion/noticia/2016/01/05/1135147/5-aplicaciones-imprescindibles-estudiantes-criminologia.htm
}

(c) EY-NC-ND 2017, Universitat Politècnica de València 
En el primer grupo destacan, entre otras, Quicktapsurvey y SurveyMonkey. Se trata de dos aplicaciones que permiten diseñar, administrar y gestionar una encuesta, tanto en modo online como offline. En el gráfico inferior se observa un ejemplo de una interfaz de este tipo de apps. Con carácter general, el usuario puede crear, elegiendo diversos modelos y contenidos, cada pantalla del cuestionario. De igual forma, como se aprecia en la parte derecha de la imagen, la app permite analizar los resultados obtenidos de 300 encuestados sobre un ítem en particular de forma automática.

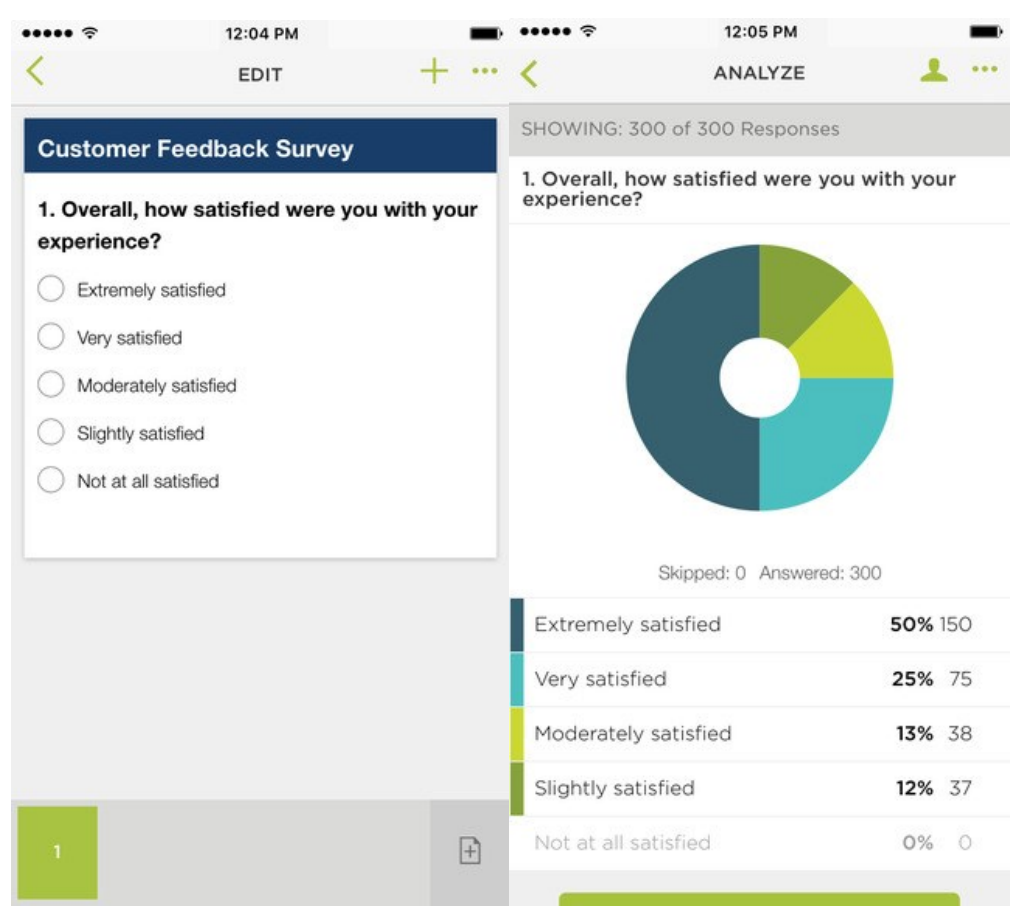

Fig. 1 Apps genéricas para el diseño de encuestas.

En esta línea, aunque ciñéndose únicamente a las propias percepciones de un sujeto que observa la realidad -y que suele dirigir la investigación- encontramos la aplicación Observe ${ }^{5}$. Es especialmente útil para que el alumno analice mediante la observación directa o participante los fenómenos que acontecen a nuestro alrededor. Observe no solo registra a tiempo real todos los datos, sino que permite su volcado y posible comparación con la de otros alumnos que hayan realizados observaciones conjuntas.

\footnotetext{
5 En el ámbito criminológico español son inexisentes las plantillas estandarizadas que permitan sistematizar información etnográfica. A fecha de hoy se viene todavía utilizando el tradicional cuarderno de campo para realizar anotaciones. Itunes PREVIEW. STUART M.SEPP. https://itunes.apple.com/us/app/observe/id439532109? mt=8 [Consulta: 31 de mayo de 2017].
} 


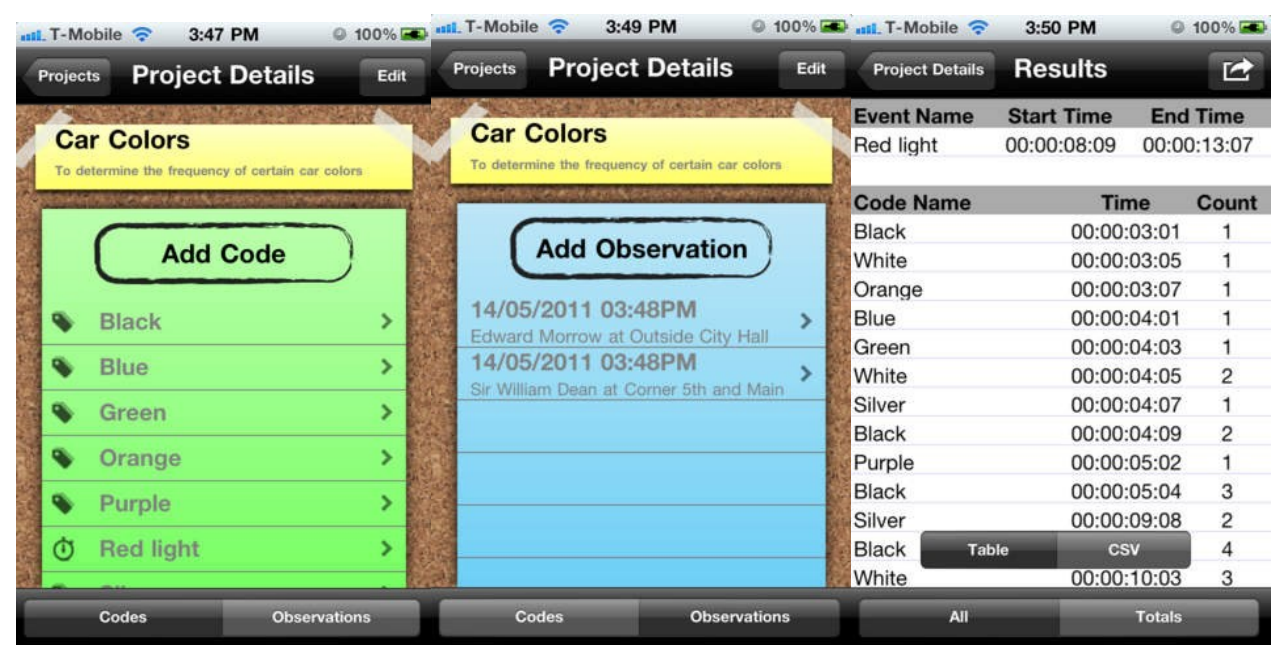

Fig. 2 Apps para la observación directa y participante.

Del segundo grupo destacan aquellas aplicaciones que hacen uso del sistema de geolocalización o GPS del dispositivo móvil con el fin de cruzar los datos provenientes de las percepciones con coordenadas temporales (horas y segundos) y espaciales (posición X e Y). Con carácter general, se emplean desde postulados teóricos situacionales que analizan y explican el fenómeno delictivo otorgando relevancia al factor ambiental, como serían los estudios con mapas del delito (Vozmediano y San Juan, 2010). o mapping crime. En este sentido, destaca la aplicación FOCA ${ }^{6}$ desarrollada por la University College de Londres. La herramienta permite recopilar datos que "proporcionan una idea de cuándo y dónde se experimenta miedo a la delincuencia, y por quién".

\footnotetext{
${ }^{6}$ FOCA es una app gratuita y disponible en Google Play. Para más información veáse: Mapping fear of crime dynamically on everyday transport.
} 


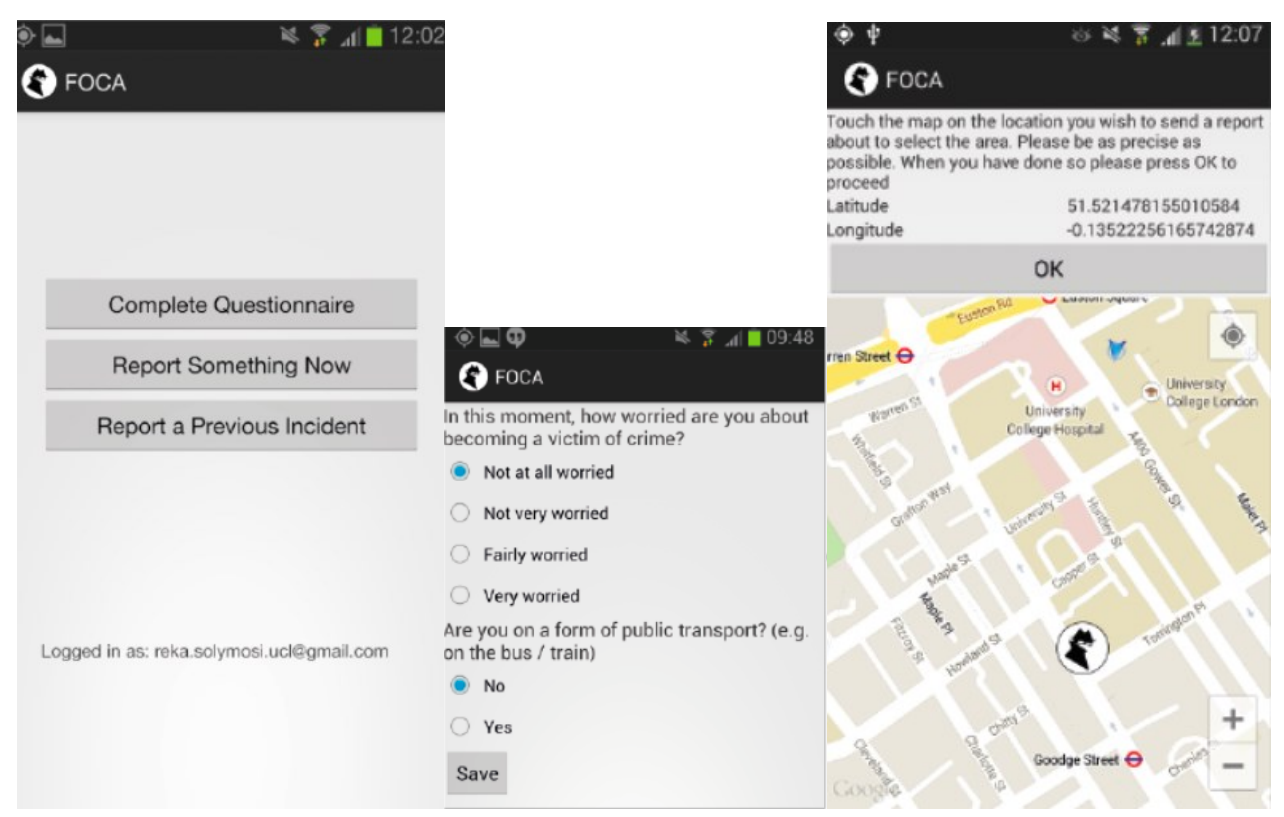

Fig. 3 Apps especializadas en medir el miedo al delito.

En la imagen superior se observan tres de las principales pantallas de la app. El sujeto puede completar un cuestionario en diversos momentos del día (mañana y noche) o bien informar sobre un suceso repentino del que ha sido víctima o testigo. En la segunda pantalla (imagen central), se estima el nivel del miedo y otras variables, como sería si se encuentra en un medio de transporte público, así como otra información adicional a determinar (sexo, edad, lugar de residencia, etc). En la tercera y última imagen (parte derecha), se muestra el mapa donde ha tenido lugar el evento que se informa.

\subsection{Ventajas y desventajas de su aplicación}

En términos generales, ambos tipos de apps -genéricas y especializadas- son muy versátiles y sirven de complemento ideal para mejorar el aprendizaje y la investigación en comparación a las herramientas tradicionales.

Principales ventajas de las apps:

- Ya están integradas en las rutinas de vida de los sujetos, especialmente de los jóvenes.

- Al tratarse de una herramienta para la investigación puede ser empleada por profesores del grado de Criminología de materias muy diversas.

- Se requiere de una escasa o nula inversión económica (el precio de la app, en su caso). 
- Su inmediatez. En cuestión de pocos días se puede finalizar un trabajo de campo que hubiese llevado meses con un cuestionario tradicional.

- La magnitud de la muestra. Las apps permiten abarcar muestras muy grandes de sujetos de diferentes cursos y clases.

- El carácter anónimo de la interacción que se produce entre el sujeto y su dispositivo móvil facilita recabar información íntima o comprometida (acoso entre iguales, agresiones sexuales sufridas, etc.).

- La recolección automática de respuestas a tiempo real. A cada momento se puede constatar los resultados de la investigación, lo que resulta de vital importancia a la hora de subsanar errores o tomar nuevas decisiones.

En cuanto a las desventajas:

- Los estudiantes o profesores que no contaran con acceso a internet en el móvil quedarían fuera de la acción.

- La ausencia de un entrevistador o figura de autoridad durante la encuesta puede provocar que se reste importancia al procedimiento o no se proporcione toda la información.

\section{Objetivos}

Con la utilización de las apps de recolección de datos se podrían alcanzar los siguientes propósitos:

2.1. Crear y administrar cuestionarios 2.0, así como tratar los datos recabados y su posterior presentación en clase. Por ejemplo, los alumnos podrían desarrollar proyectos para estimar la victimización sufrida (en delitos sexuales, violencia de género, robos, etc) y medir el miedo al delito en función del lugar de residencia y rutinas de vida, entre otros.

2.2. Sistematizar los datos de la observación directa o participante.

2.3. Diseñar nuevas apps especializadas o proyectos de investigación cuya metodología se fundamente en la recolección de datos cualitativos provenientes de percepciones de sujetos.

\section{Desarrollo de la innovación}

El empleo de la app se llevaría a cabo con estudiantes del grado de Criminología cuya edad media es inferior a 21 años de edad, por lo que estos nativos digitales ${ }^{7}$ poseerían

\footnotetext{
${ }^{7}$ Los jóvenes menores de veinte años son sus mayores representantes. Estos han crecido inmersos en un continuo bombardeo digital y han integrado la tecnología en su aprendizaje más temprano (Small y Vorgan, 2009). La mente joven en desarrollo codifica la información de forma diferente a como lo haría un adulto al tiempo que su atención está más limitada y focalizada en
} 
un smartphone y estarían muy familiarizados con las TICs. Así mismo, no tendrían necesidad de desplazarse para realizar el trabajo de campo siendo suficiente la mera instalación de la app en el móvil. Dependiendo de la asigntaura y del curso, cada profesor podría optar por desarrollar los objetivos que pretende alcanzar aplicando una de las apps anteriormente mencionadas. De igual forma, cabría su administración de forma simultánea en diversos grupos y cursos académicos.

\subsection{Planteamiento de la actividad}

Los dos primeros objetivos de la acción docente se desarollarían a modo de tarea teórico-práctica requiriendo del compromiso y participación del alumno tanto en clase como en el trabajo de campo. Si se trata de la creación y administración de un cuestionario relativo a la propia victimización sufrida, se debe incentivar su participación haciendo especial hincapié en la relevancia de la investigación al tiempo que se recalca su carácter anónimo y confidencial. En el caso de ejecutar una observación directa o participante, se debe plantear la actividad en diferentes contextos sociales para que el alumno sea más versátil a la hora de discriminar qué aspectos de la realidad ha de registrar.

\subsection{Formación}

El primer paso para desarrollar la innovación es la formación del alumno en aplicaciones de software y nuevas tecnologías. A pesar de que se presupone que los estudiantes mantienen una relación natural con las mismas, es necesario explicar el funcionamiento y potencialidades de las apps de recolección de datos, la elección de la más adecuada dentro de las ofertadas y el tratamiento de los datos. Así mismo, y dependiendo de la formación metodológica del grupo en concreto, sería necesario administrar conocimientos sobre diseño de cuestionarios y selección de contactos clave ${ }^{8}$. De igual forma, si está desempeñando una observación directa o participante, se le ha de instruir para que identifique y registre en el dispositivo todas aquellas situaciones de la vida cotidiana. Por último y, con referencia al objetivo 2.3, se deben reforzar las habilidades del alumno en la gestión y búsqueda de recursos bibliográficos relacionados con las nuevas tecnologías, y en su capacidad para proyectar soluciones digitales a necesidades reales.

\footnotetext{
varios estímulos a la vez. Por ello, la televisión es percibida como "lenta y aburrida" o un libro "una cadena de palabras, sosa e inmutable". Por el contrario, el ciberespacio contenido en el smartphone que guardan en el bolsillo les conecta con el mundo exterior, con sus amigos y, en definitiva, con todas aquellas formas de ocio que brinda internet. Así, lo habitual es que el joven vea la televisión mientras recibe wasaps, consulta el correo electrónico, juega on-line o lee un libro. Los adultos, sobre todo aquellos que rondan los 40 años de edad, también están inmersos en la era digital, sin embargo se consideran inmigrantes digitales (Small y Vorgan, 2009) al pasar menos tiempo expuestos a las tecnologías.

8 En la observación participante, como en cualquier otra metodología que requiera del acceso y contacto a un grupo determinado, es esencial que el alumno esté formado en competencias que posibiliten recabar información sobre los sujetos, sus costumbres, lugares de reunion, disposición a ser contactados, etc. (Álvarez, 2008).
}

(c) $)$ EY-NC-ND 2017, Universitat Politècnica de València 


\subsection{Puesta en marcha de la actividad}

Una vez impartida la formación comienza la tarea práctica. Si la app es genérica, los alumnos deberán emplear varias sesiones en diseñar el cuestionario, por el contrario, si se opta por una app especializada, tan solo será necesario su mera descarga. En cuanto a las tareas vinculadas al objetivo tercero (2.3), la parte práctica daría comienzo con exposiciones individuales o grupales sustentadas en dinámicas que generen debates sobre las ideas innovadoras.

\subsection{Seguimiento de la actividad}

Al igual que cualquier otra tarea, se debe temporalizar su ejecución fijando una fecha de inicio y final. En el caso de que sea el alumno quien haya creado y gestionado su propio cuestionario, se debe contar con el control y apoyo externo del profesor, sobre todo si surgen problemas que puedan comprometer la recogida de datos o la ejecución del trabajo de campo. En este sentido, se debe apostar por un sistema de evaluación continua que comprenda la evaluación periódica da datos. Si la tarea consiste en el diseño de nuevas apps especializadas o proyectos de investigación, el seguimiento debería enfatizar la planificación de un calendario prolongado de exposiciones.

\subsection{Análisis y tratamiento de la información}

Una de las principales ventajas de las apps es la recolección rápida de grandes cantidades de información. En este sentido, es esencial que el alumno sepa determinar la calidad de la misma y si ha sido correctamente grabada. Un vez tratada la información, la misma puede utilizarse para aprender a redactar un informe con datos empíricos y preparar una exposición de resultados, competencias vinculadas a la elaboración final del trabajo fin de grado.

\section{Resultados}

Los impactos de la innovación docente se traducen en la adquisición de nuevas competencias por parte del alumnado y el profesorado.

\subsection{En el alumnado:}

1. Desarrollar nuevas habilidades en el campo de las TICs más allá del manejo de las herramientas 2.0 habituales (campus virtual y correo electrónico).

2. Aprender a plantear, diseñar y ejecutar una investigación empírica empleando herramientas metodológicas 2.0 .

2017, Universitat Politècnica de València 
3. Mejorar sus competencias relacionales con contactos clave de los contextos a investigar, esto es, pautas de presentación, argumentación y persuasión.

4. Analizar y tratar grandes cantidades de datos estadísticos provenientes de apps.

5. Redactar informes y mejorar esta competencia de cara al trabajo fin de grado.

6. Mejorar la expresión oral y las dotes comunicativas para transmitir ideas innovadoras.

7. Recibir formación en competencias específicas (geolocalización o mapping, victimología, etc.).

8. Diseñar intervenciones eficaces y eficientes para prevenir el delito una vez analizados los datos. Por ejemplo: elaboración de diagnósticos medioambiantales en determinadas calles, barrios o zonas con el fin de elaborar propuestas de prevención situacional, implementar estrategias que reduzca determinados tipos de delincuencia no denunciada y la percepción de seguridad, etc.

\subsection{En el profesorado:}

1. Desarrollar nuevas habilidades en el campo de las TICs más allá del manejo de las herramientas 2.0 habituales (campus virtual y correo electrónico).

2. Utilizar para la acción formativa el diseño y ejecución de investigaciones empíricas empleando herramientas metodológicas 2.0.

3. Mejorar la acción formativa práctica al elaborar estudios empíricos y reales con carácter previo o en el trabajo fin de grado.

4. Conseguir un mayor grado implicación y atención del alumno en comparación a las tareas tradicionales.

\section{Conclusiones}

Las aplicaciones de software en dispositivos móviles con acceso a internet ofrecen grandes ventajas y oportunidades para desarrollar acciones de innovación docente. El hecho de que se trate de una herramienta ampliamente utilizada por los jóvenes propicia su fácil integración en las tareas al tiempo que resulta llamativa y original. En este nuevo marco 2.0 es necesario una implicación activa del profesorado en nuevas tecnologías capaces de revolucionar el mundo de la docencia. Dicha implicación debe ser constante y adaptada a los continuos avances científicos que modifican la vida cotidiana. En este orden de ideas, no parece posible desvincular la acción formativa de la utilización de las TIC, ni tampoco preparar profesionalmente a los estudiantes si no obtienen de estas una utilidad alternativa al propio ocio y entretenimiento. En definitiva, se hace imprescindible elaborar y ejecutar propuestas docentes adaptadas a los nuevos tiempos. 


\section{Referencias}

Álvarez Álvarez, C. (2008). "La etnografía como modelo de investigación en educación", en Gaceta de Antropología, vol. 24 (1), 10, p. 1-15.

García, B., Welford, J. y SMith, B. (2015). "Using a Smartphone app in qualitative research: the good, the bad and the ugly", en Qualitative Research, 3, p. 508-525.

SMALl, G. Y Vorgan, G. (2009). El cerebro digital: cómo las nuevas tecnologías están cambiando nuestra mente. Barcelona: Editorial Urano.

Solymosi, R. (2014). Mapping fear of crime dynamically on everyday transport. London: UCL Jill Dando Institute of Security and Crime Science.

SugIE, N. (2016). "Utilizing Smartphones to study disadvantaged and hard-to-reach groups", en Sociological Methods Reseach, 18, p. 2-34.

VozMEdiAnO SANZ, L. Y SAN JuÁN GUILLÉn, C. (2010). Criminología ambiental: Ecología del delito y de la seguridad. Barcelona: Editorial UOC.

\section{Páginas webs:}

ANÁLISIS DE LAS MEJORES APPS IOS Y ANDROID PARA LA EDUCACIÓN. EDUCA APPS. http://www.educapps.es. [Consulta: 31 de mayo de 2017].

ITUNES PREVIEW. STUART M.SEPP. https://itunes.apple.com/us/app/observe/id439532109?mt=8 [Consulta: 31 de mayo de 2017].

FOCA. REKA SOLYMOSI UCL.

https://play.google.com/store/apps/details?id=uk.ac.ucl.uctzrso.foca\&hl=es. [Consulta: 31 de mayo de 2017].

QUICKTAPSURVEY. http://www.quicktapsurvey.com/lg/es/ [Consulta: 31 de mayo de 2017].

SURVEYMONKEY. https://es.surveymonkey.com/mp/iphone-survey-app/ [Consulta: 31 de mayo de 2017].

UNIVERSIA ESPAÑA. http://noticias.universia.es/educacion/noticia/2016/01/05/1135147/5aplicaciones-imprescindibles-estudiantes-criminologia.html. [Consulta: 31 de mayo de 2017]. 